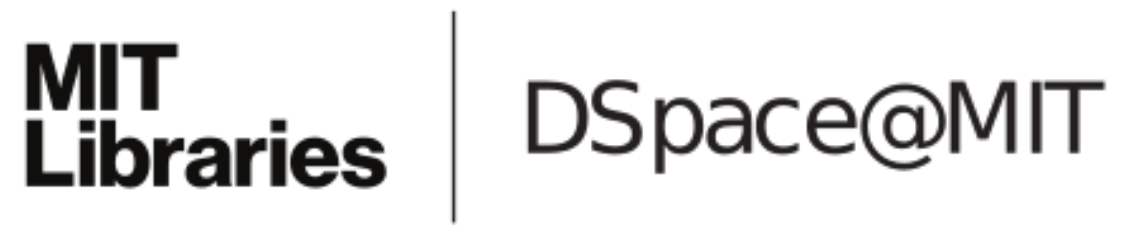

\author{
MIT Open Access Articles
}

\begin{abstract}
Gold nanoprisms as a hybrid in vivo cancer theranostic platform for in situ photoacoustic imaging, angiography, and localized hyperthermia
\end{abstract}

The MIT Faculty has made this article openly available. Please share how this access benefits you. Your story matters.

Citation: Bao, Chenchen, João Conde, Fei Pan, Chao Li, Chunlei Zhang, Furong Tian, Shujing Liang, Jesus M. de la Fuente, and Daxiang Cui. "Gold Nanoprisms as a Hybrid in Vivo Cancer Theranostic Platform for in Situ Photoacoustic Imaging, Angiography, and Localized Hyperthermia." Nano Research 9, no. 4 (February 24, 2016): 1043-1056.

As Published: http://dx.doi.org/10.1007/s12274-016-0996-y

Publisher: Tsinghua University Press

Persistent URL: http://hdl.handle.net/1721.1/105543

Version: Author's final manuscript: final author's manuscript post peer review, without publisher's formatting or copy editing

Terms of Use: Article is made available in accordance with the publisher's policy and may be subject to US copyright law. Please refer to the publisher's site for terms of use. 


\title{
Gold nanoprisms as a hybrid in vivo cancer theranostic platform for in situ photoacoustic imaging, angiography, and localized hyperthermia
}

\author{
Chenchen Bao ${ }^{1}$, João Conde ${ }^{2,3}$, Fei Pan ${ }^{1}$, Chao Li ${ }^{1}$, Chunlei Zhang ${ }^{1}$, Furong Tian ${ }^{4}$, Shujing Liang ${ }^{1}$, \\ Jesus M. de la Fuente ${ }^{1,5}$, and Daxiang Cui ${ }^{1}(\varangle)$ \\ ${ }^{1}$ Institute of Nano Biomedicine and Engineering, Department of Instrument Science and Engineering, School of Electronic Information and \\ Electrical Engineering, National Center for Translational Medicine, Collaborative Innovational Center for System Biology, Shanghai Jiao \\ Tong University, Shanghai 200240, China \\ ${ }^{2}$ Massachusetts Institute of Technology, Institute for Medical Engineering and Science, Harvard-MIT Division for Health Sciences and \\ Technology, E25-449 Cambridge, Massachusetts, USA \\ ${ }^{3}$ School of Engineering and Materials Science, Queen Mary University of London, E1 4NS London, UK \\ ${ }^{4}$ School of Food Science and Environmental Health, College of Science and Health, Dublin Institute of Technology, Camden Row, 4 \\ Dublin, Ireland \\ ${ }^{5}$ Instituto de Nanociencia de Aragon (INA), Universidad de Zaragoza, Zaragoza 50018, Spain
}

Received: 17 October 2015

Revised: 20 December 2015

Accepted: 3 January 2016

(C) Tsinghua University Press and Springer-Verlag Berlin Heidelberg 2016

\section{KEYWORDS}

gold nanoprisms, in situ gastric cancer, photoacoustic imaging, photothermal therapy

\begin{abstract}
The development of high-resolution nanosized photoacoustic contrast agents is an exciting yet challenging technological advance. Herein, antibody (breast cancer-associated antigen 1 (Brcaa1) monoclonal antibody)- and peptide (RGD)functionalized gold nanoprisms (AuNprs) were used as a combinatorial methodology for in situ photoacoustic imaging, angiography, and localized hyperthermia using orthotopic and subcutaneous murine gastric carcinoma models. RGD-conjugated PEGylated AuNprs are available for tumor angiography, and Brcaa1 monoclonal antibody-conjugated PEGylated AuNprs are used for targeting and for in situ imaging of gastric carcinoma in orthotopic tumor models. In situ photoacoustic imaging allowed for anatomical and functional imaging at the tumor site. In vivo tumor angiography imaging showed enhancement of the photoacoustic signal in a time-dependent manner. Furthermore, photoacoustic imaging demonstrated that tumor vessels were clearly damaged after localized hyperthermia. This is the first proof-of-concept using two AuNprs probes as highly sensitive contrasts and therapeutic agents for in situ tumor detection and inhibition. These smart antibody/peptide AuNprs can be used as an efficient nanotheranostic platform for in vivo tumor detection with high sensitivity, as well as for tumor targeting therapy, which, with a single-dose injection, results in tumor size reduction and increases mice survival after localized hyperthermia treatment.
\end{abstract}

Address correspondence to dxcui@sjtu.edu.cn 


\section{Introduction}

Since Richard Feynman's famous talk, "There's plenty of room at the bottom" at an American Physical Society meeting at Caltech in 1959 [1], nanotechnology has led to the development of new materials (e.g. nanoparticles (NPs)) and devices [2] with a wide-range of applications, especially in imaging, diagnostics, and therapy, contributing to the early detection and treatment of cancer and metastasis $[3,4]$. NPs are within the same size domain as many biomolecules, including enzymes, antibodies, and protein receptors. This, combined with the unique properties of materials in the nanosize range, provides scope for improved biomaterials for therapy and diagnostics.

Since light absorption from biological tissue components is minimized at near-infrared (NIR) wavelengths, most NPs for in vivo imaging have been designed to strongly absorb in the NIR region so as to be effective contrast agents [5, 6]. Gold-nanoprisms (AuNprs) have been extensively used in bioimaging owing to their ability to tune the plasmon resonance maximum further into the NIR region $[7,8]$. More recently, Bao et al. reported the use of AuNprs as a novel contrast agent for the hybrid technique of photoacoustic imaging (PAI). The authors demonstrated an in silico electron tomographic reconstruction of such Au nanostructures, which showed promise for application in biomedical imaging, drug delivery, and photothermal therapy [9].

Light, as an electromagnetic wave, possesses the property of diffraction and diffusion, which inevitably confines its application in bioimaging of tumors. Localized imaging with both deep penetration and high resolution is still a challenge for other modalities such as optical imaging and ultrasound imaging. In PAI, a pulse of NIR laser light is used in resonance with the surface plasmon instead of a continuous NIR source. This technique causes rapid thermal expansion of the surrounding media and the generation of a sound wave that can be detected on the surface of the subject. The use of NIR reduces the amount of absorption that occurs by the light, however absorption of the light by various other organs is unavoidable [10].

Numerous systems using $\mathrm{Au}$ nanocages [11] or Au nanorods $[12,13]$ have been used as NIR contrast- enhancing agents for photoacoustic tomography and to image their distribution when circulating in the vasculature of mice tissues, as well as to enhance the diagnostic power of optoacoustic imaging. Nevertheless, a dual AuNprs probe used as a combinatorial platform for photoacoustic angiography, localized hyperthermia using a subcutaneous, and an orthotopic murine gastric carcinoma model has never been reported so far.

Although it is not a replacement for traditional surgery, radiotherapy, or chemotherapy, localized hyperthermia has been used in numerous animal tumor studies, producing significant synergies with a complementary role for treating cancer [14, 15]. Recently, Ambrosone et al. reported the use of hyperthermia using naked $\mathrm{Au}$ triangles that caused hyperthermia overexpression of heat shock protein hsp70 with concomitant cell necrosis in an invertebrate animal model (Hydra vulgaris) [8]. More recently, Han et al. reported glucose-functionalized AuNprs for photothermal therapy of cancer tissue [16]. However, the impact on the cancer cells and the structure, function, growth, and metabolism caused by a series of genetic changes using $\mathrm{Au}$ triangles in higher organisms remains unknown.

Here, we report that RGD conjugated PEGylated (PEG/RGD)-AuNprs are available for tumor angiography and breast cancer-associated antigen 1 (Brcaa1) monoclonal antibody-conjugated PEGylated (PEG/ Brcaa1)-AuNprs are used for targeting and in situ imaging of gastric carcinoma in orthotopic tumor models with high resolution and efficiency rates (Fig. 1). In situ photoacoustic imaging allowed for high resolution of blood vessels at the tumor site, with an enhancement of the photoacoustic signal in a timedependent manner. This platform is the first proofof-concept, reporting two AuNprs probes used as high sensitivity contrasts and therapeutic agents for in situ PAI of gastric cancer, in vivo tumor detection, and inhibition. These smart and biocompatible PEG/RGDAuNprs and PEG/Brcaa1-AuNprs can be used as efficient and safe nanotheranostic platforms for high sensitivity in vivo tumor detection, as well as for tumor targeting, which results in tumor size reduction and increases mice survival after localized hyperthermia treatment. 

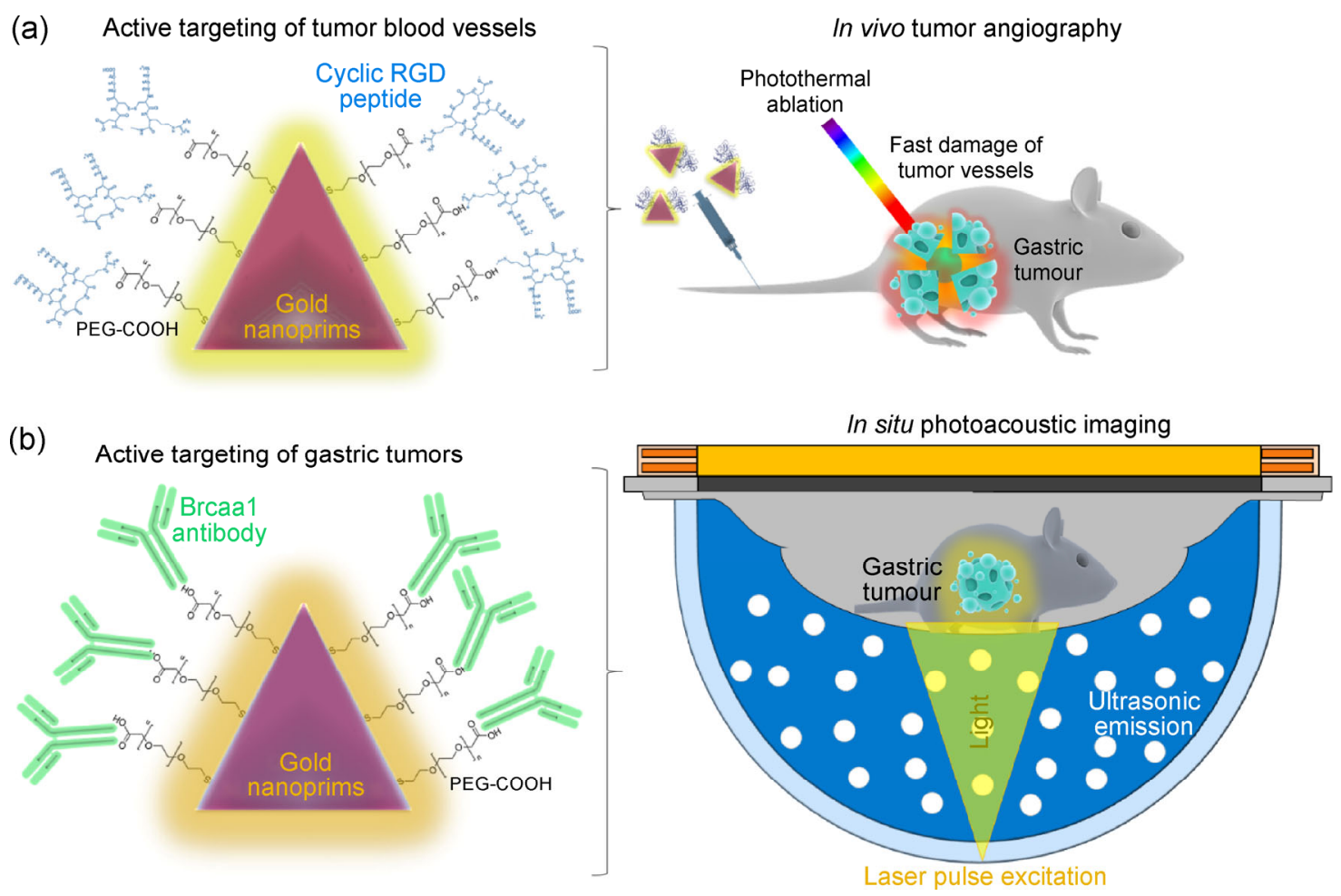

Figure 1 AuNprs as a hybrid in vivo cancer theranostic platform for in situ photoacoustic imaging, angiography, and localized hyperthermia. (a) Schematic illustration of the AuNprs functionalized with PEG and a cyclic RGD peptide (PEG/RGD-AuNprs) and their application in photoacoustic angiography and in targeted phototherapy in the tumor vessels. (b) Schematic illustration of the antibody Brcaa1-AuNprs and their application in in situ photoacoustic imaging of gastric tumors.

\section{Experimental}

\subsection{Synthesis and characterization of functionalized PEGylated AuNprs}

The surfactant-free methods for synthesis and characterization of functionalized PEGylated AuNprs can be found in our previous publications $[7,9]$. Briefly, before functionalization, AuNprs were washed in deionized (DI) water and suspended in 2(morpholino)ethanesulfonic acid (MES; 0.1 M, pH 6.0) solution. 3-(3-Dimethylaminopropyl)-carbodiimide (EDC) and N-hydroxysulfosuccinimide (sulfo-NHS) were added to $300 \mu \mathrm{L}$ of the PEGylated AuNpr solution in the reactive MES solution $(0.1 \mathrm{M}, \mathrm{pH}=6)$ and allowed to react for $30 \mathrm{~min}$ at room temperature with EDC and sulfo-NHS final concentrations of 40 and $35 \mathrm{mM}$, respectively. After the reaction, the buffer was replaced by phosphate-buffered saline (PBS, $\mathrm{pH}=$ 8.0) and an excess of RGD-4C (GL Biochem Shanghai Ltd, China) or $300 \mu \mathrm{L}$ Brcaa1 [17] monoantibody
( $2 \mathrm{mg} / \mathrm{mL}$ ) was added and incubated for $3 \mathrm{~h}$ at room temperature. The resulting PEGylated AuNprs were stored in the dark at $4{ }^{\circ} \mathrm{C}$ until further use.

The absorbance spectra of AuNprs, PEG-AuNprs, and RGD-PEG-AuNprs were measured by a Varian Cary 50 UV-Vis spectrophotometer. The morphology of the AuNprs was evaluated using high-resolution transmission electron microscopy (TEM, JEOL, JEM2010) and field emission scanning electron microscopy (FESEM, Zeiss Ultra5). The photoacoustic signal acquisition was performed using 10 replicates at each wavelength $(680,700,710,808$, and $950 \mathrm{~nm})$ in a Nexus 128 (Endra Nexus 128, USA) and analyzed by OsiriX imaging software (OsiriX Foundation, Genève, Switzerland).

\subsection{In vitro study of RGD-PEG-AuNprs}

Human gastric epithelial mucosa cell line, GES-1, and human gastric cancer cell line, MGC-803, were used as models. GES-1 cells were cultured in a $5 \% \mathrm{CO}_{2}^{-}$ 
balanced air incubator at $37^{\circ} \mathrm{C}$ in Dulbecco's modified Eagle's medium (DMEM) containing 10\% fetal bovine serum (FBS) and 1\% penicillin-streptomycin. MGC-803 cells were cultured in RPMI 1640 medium containing $10 \% \mathrm{FBS}$ and $1 \%$ penicillin-streptomycin at $37{ }^{\circ} \mathrm{C}$ and in $5 \% \mathrm{CO}_{2}$. GES-1 cells were first cultured to $60 \%$ confluency before trypsinized to 5,000/well and seeded in a 96-well cell culture plate. Cells were incubated overnight in a conventional cell culture environment. For the MTT assay, cells were incubated with 50, 100, 150, and $200 \mu \mathrm{g} / \mathrm{mL}$ of naked AuNprs, PEG-AuNprs, RGD-PEG-AuNprs, or AuNprs-Brcaa1 particles for $24 \mathrm{~h}$. The absorbance was read at $570 \mathrm{~nm}$ and the cell viability was determined from the intensity ratio of the treated to non-treated control cells and shown as an average \pm standard deviation $(n=5)$.

For the TEM images, MGC-803 cells were incubated with RGD-AuNprs (50 $\mu \mathrm{g} / \mathrm{mL} \mathrm{Au})$ for $24 \mathrm{~h}$ and collected. The cells were treated with $2.5 \%$ glutaraldehyde phosphate buffer and fixed overnight before being rinsed with $0.1 \mathrm{M}$ PBS three times (15 min each), incubated in $1 \%$ osmium tetroxide for $2 \mathrm{~h}$, and rinsed with $0.1 \mathrm{M}$ phosphate three times (15 min each). Samples were dehydrated sequentially in $30 \%$ and $50 \%$ ethanol for $10 \mathrm{~min}, 70 \%$ ethanol containing $2 \%$ uranyl acetate, and finally stained overnight. Next, the samples were dehydrated in $70 \%$ and $90 \%$ ethanol for $10 \mathrm{~min}$ each, followed by incubation in a $90 \%$ acetone/90\% ethanol $(1: 1 \mathrm{v} / \mathrm{v})$ solution for $15 \mathrm{~min}$ and rinsed in $90 \%$ acetone for $15 \mathrm{~min}$. Finally, the samples were rinsed with $100 \%$ acetone at room temperature three times, embedded with +812 epoxy with pure acetone $(1: 1 v / v)$ at room temperature for $1-2 \mathrm{~h}$, followed by pure acetone +812 epoxy resin $(1: 2)$ at room temperature for 3-4 h, and finally embedded in epoxy resin overnight with 812 . The following day, the samples were embedded in epoxy resin 2-3 times every $4 \mathrm{~h}$. The samples were cured at $37^{\circ} \mathrm{C}$ overnight, then $45^{\circ} \mathrm{C}$ for $12 \mathrm{~h}$, and $60{ }^{\circ} \mathrm{C}$ for $48 \mathrm{~h}$. With a microtome slice thickness of $70 \mathrm{~nm}$ and lead citrate staining, the samples were imaged for TEM (JEOL, JEM-2010).

For cell viability after laser irradiation, MGC-803 cells were incubated with RGD-AuNprs for $24 \mathrm{~h}$. Then, the cells were washed twice with PBS and irradiated with $980 \mathrm{~nm}$ laser light for $3 \mathrm{~min}$ at $2 \mathrm{~W} / \mathrm{cm}^{2}$. After irradiation, the cells were stained with Trypan Blue.
The control group was MGC-803 cells without NP treatment.

\subsection{Construction of murine gastric cancer models}

All animal experiments were approved by the Institutional Animal Care and Use Committee of Shanghai Jiao Tong University (NO.SYXK2007-0025). Female nude mice (nu/nu, $18 \mathrm{~g}$, and 4 weeks old) were purchased from the Shanghai SLAC Laboratory Animal Co. Ltd and housed in an SPF grade animal center. Subcutaneous gastric cancer (GC) tissues at the exponential growth phase loaded in nude mice were resected aseptically. Necrotic tissues were cut away, and the remaining tumor tissues were scissor-minced into pieces around 1-2 $\mathrm{mm}$ in diameter in $4{ }^{\circ} \mathrm{C}$ Hanks' balanced salt solution and each piece was adjusted to $30 \mathrm{mg}$ using scissors. Before the surgery, all mice were fasting for at least $8 \mathrm{~h}$. Mice were anesthetized with $5 \%$ trichloraldehyde hydrate $(4 \mu \mathrm{L} / \mathrm{g}$ weight $)$ and an incision was made through the left upper abdominal pararectal line and peritoneum. The stomach wall was carefully exposed, and a part of the serosal membrane $(\sim 2 \mathrm{~mm}$ in diameter) in the middle of the greater curvature of the glandular stomach was mechanically scratched with ophthalmic knives. A tumor piece of $30 \mathrm{mg}$ was then fixed on the injured site of the serosal surface with OB glue (Guang Zhou Baiyun Ltd, China). The stomach was then returned to the peritoneal cavity, and the abdominal wall and skin were closed with 9-0 ophthalmic sutures. The mice were kept on a warm electric blanket at $37^{\circ} \mathrm{C}$ until analepsis and kept separately in an SPF environment.

\subsection{In vivo PAI study of murine gastric cancer models}

In vivo PAI was accomplished by a PA system (Endra Nexus 128, USA) with the excitation laser set at the bottom of a hemispherical bowl. For subcutaneous tumor models, mice were injected intraperitoneally with $5 \%$ chloral hydrate $(w / v)$ and supplemented with $1 \%-2 \%$ isoflurane, an appropriate gas for anesthesia. Nude mice with GC xenografts (subcutaneous or orthotopic models) were intravenously injected with $100 \mu \mathrm{L}$ of $1 \mathrm{mg} / \mathrm{mL}$ RGD-AuNprs or Brcaa1-AuNprs, respectively. The Nexus 128 system was equipped with 
high (for subcutaneous xenografts) or low positions (for orthotopic xenografts) for image acquisition. The following irradiation laser wavelengths were chosen to observe the in vivo PA signal of the xenografts: 680 , $700,710,808,950$, and $710 \mathrm{~nm}$. Data were collected after 1,3 , and $6 \mathrm{~h}$. The PA signals, which were received by the ultrasonic transducers, were spirally distributed on the surface of the bowl before finally directed to computational graphic reconstruction. Reconstruction of the 2D and 3D PA images was performed by OsiriX imaging software (OsiriX Foundation, Switzerland).

\subsection{NIR absorption digital high-definition infrared} microscopic imaging and localized hyperthermia of murine subcutaneous tumor model of gastric cancer

For in vitro measurements, the photothermal effect of AuNprs was evaluated via irradiation by a $980 \mathrm{~nm}$ laser (LOS-BLD-0980-1.3W-C, Hi-Tech Optoelectronics Co., Ltd. China) and read by an oscillometer (34970A, Agilent, USA). Briefly, for in vivo applications, a trial group (GC xenograft mice transplanted subcutaneous tumor models) was injected with $100 \mu \mathrm{L}$ of $1 \mathrm{mg} / \mathrm{mL}$ RGD-AuNprs via tail vein. After $2 \mathrm{~h}$, the $980 \mathrm{~nm}$ laser was applied to irradiate the tumor site and the images of temperature variation at the tumor site were accomplished by the digital high-definition infrared microscopic imaging system (Ying Fu Photoelectric Technology Co. Ltd, Shanghai, China). The laser energy was $2 \mathrm{~W} / \mathrm{cm}^{2}$ (read according to a standard curve, data not shown).

\subsection{Computed tomography (CT) imaging}

In order to compare with the PAI, nude mice with GC xenografts (subcutaneous or orthotopic models) were also intravenously injected with $100 \mu \mathrm{L}$ of $1 \mathrm{mg} / \mathrm{mL}$ RGD-AuNprs and Brcaa1-AuNprs and used in CT and X-ray imaging. CT imaging (Latheta LCT200, Hitachi-Aloka, Japan) was used for in situ imaging with the tube voltage of $50 \mathrm{kVp}$ and exposure time of $300 \mathrm{~ms}$. For X-ray imaging (Carestream FX PRO, KODAK, USA), the exposure time was $15 \mathrm{~s}$.

\subsection{Distribution and pathological analysis of RGD- PEG-AuNprs in subcutaneous gastric cancer models}

For in situ models, tumors were removed $24 \mathrm{~h}$ after the injection of Brcaa1-AuNprs and fixed in 5\% paraformaldehyde overnight before being embedded in paraffin for tissue sections. For immunofluorescence staining, Brcaa1 mouse anti-human antibody $(1 \mathrm{mg} / \mathrm{mL}$ concentration of 1:100) was used as the primary antibody and FITC-rabbit anti-mouse (Abcam, UK) as the secondary antibody $(1: 1,000)$.

For in vivo biodistribution studies, nude mice with GC xenografts were intravenously injected with $100 \mu \mathrm{L}$ of $1 \mathrm{mg} / \mathrm{mL}$ RGD-AuNprs before being sacrificed at $24 \mathrm{~h}$ and 6 weeks post-injection ( $n=3$ /group). The heart, liver, spleen, lungs, kidneys, and tumor sites were collected and weighed. The tissues $(50 \mathrm{mg}$ ) were digested with aqua regia under heating. The Au concentration in solution was determined by an iCAP 6000 Radial (Thermo, USA).

\subsection{Statistical analysis}

Each experiment was repeated three times in duplicate if not stated otherwise. The results were presented as the mean \pm SD. Statistical differences were evaluated using the $t$-test and considered significant at $P<0.05$.

\section{Results and discussion}

In this study, antibody (Brcaa1 monoclonal antibody) and peptide (RGD) functionalized AuNprs were used as a combinatorial methodology for in situ photoacoustic imaging, angiography, and localized hyperthermia for gastric carcinoma. RGD peptides have been extensively investigated as cell adhesion peptides that are recognized by cell-surface receptors, such as integrin $\alpha v \beta 3$. RGD peptide can mediate cell adhesion and proliferation and plays an important role in tumor angiogenesis and metastasis, being considerably upregulated in the endothelium during angiogenesis. Therefore, RGD represents a marker for malignancy [18] and has become a useful tool for the targeting of drugs and probes for functionalized imaging contrast agents $[19,20]$.

Brcaa1 was screened from a large number of clinical specimens associated with the breast cancer antigen gene. It was also demonstrated to have high expression in $65 \%$ of gastric cancer cells, and this expression increases with the progression of malignancy [17]. Therefore, the Brcaa1 monoclonal antibody was 
prepared and applied to target human gastric cancer cells [21, 22].

AuNprs coated with PEG-COOH, a cyclic peptide RGD-4C (Arg-Gly-Asp bicyclic peptide, ACDCRGDCFCG, disulfide bonds), and an antibody for Brcaa1 were characterized by TEM and UV-visNIR spectroscopy extinction. Figure 2(a) represents the TEM image of naked AuNprs. A nearly flat shape of an equilateral triangle with a side length of about $110 \mathrm{~nm}$ can be observed. UV-vis-NIR spectroscopy extinction spectra of naked RGD-conjugated PEGylated AuNprs are depicted in Fig. 2(b). Two distinct peaks around 980 and $530 \mathrm{~nm}$ can be observed with a slightly red-shifted peak for RGD-conjugated PEGylated AuNprs.

In order to normalize the AuNprs photoacoustic signals as a function of the concentration, the lowest concentration of AuNprs was selected as an internal reference solution, with an absorbance of approximately 0.05 OD. A linear relationship $\left(R^{2}=0.991\right)$ between the absorption at $710 \mathrm{~nm}$ and the concentration of the photoacoustic signal in relation to the AuNprs hemispherical photoacoustic detection system is represented in Fig. 2(c).

To evaluate the thermal effects of AuNprs, the temperature of AuNprs in aqueous solution was evaluated over time using a 980-nm laser light source (Agilent digital thermocouple and logger detection) (Fig. 2(d)). Figure 2(d) shows the variation of temperature under $980 \mathrm{~nm}$ laser irradiation near the heating location $\sim 1 \mathrm{~cm}$ from the area under laser illumination. Six different concentrations of AuNprs (0,5, 10, 30, 75, 150, and $300 \mu \mathrm{g} / \mathrm{mL}$ ) were applied. The temperature of the AuNprs solution increases to around $88{ }^{\circ} \mathrm{C}$ for the $300 \mu \mathrm{g} / \mathrm{mL}$ AuNprs under $0.3 \mathrm{~W} / \mathrm{cm}^{2}$ irradiation for $5 \mathrm{~min}$. At a moderate AuNprs concentration of $75 \mu \mathrm{g} / \mathrm{mL}$, the $\Delta T$ reached $31^{\circ} \mathrm{C}$. These results clearly demonstrate that the prepared AuNprs have substantial thermal effects under low laser energy irradiation, which is the basis of their application as photoacoustic contrast agents and for hyperthermia treatment of cancerdiseased cells.

The cellular uptake of the AuNprs was characterized using TEM (Fig. S1 in the Electronic Supplementary Material (ESM)) and two-photon microscopy (Fig. S2 in the ESM). The TEM images show subcellular localization of the RGD-conjugated PEGylated AuNprs in MGC-803 cells (Fig. S1(a) in the ESM). RGDconjugated PEGylated AuNprs are distributed in the cytoplasm within the cell vesicles (Fig. S1(b) in the ESM). Under a $746 \mathrm{~nm}$ excitation laser, two-photon fluorescence microscopy showed that RGD-conjugated (a)

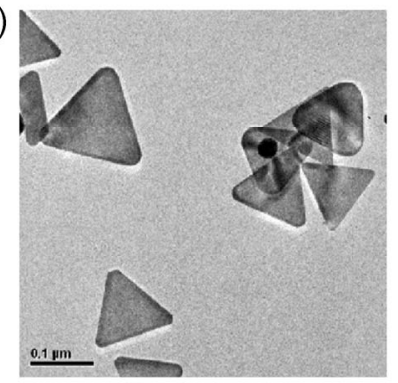

(c)

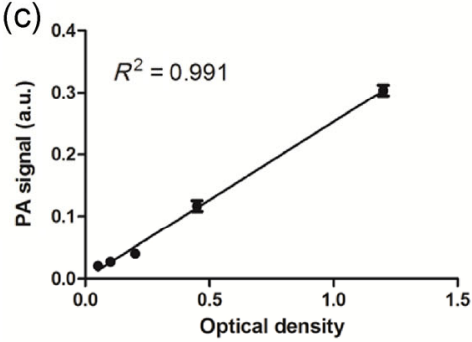

(b)

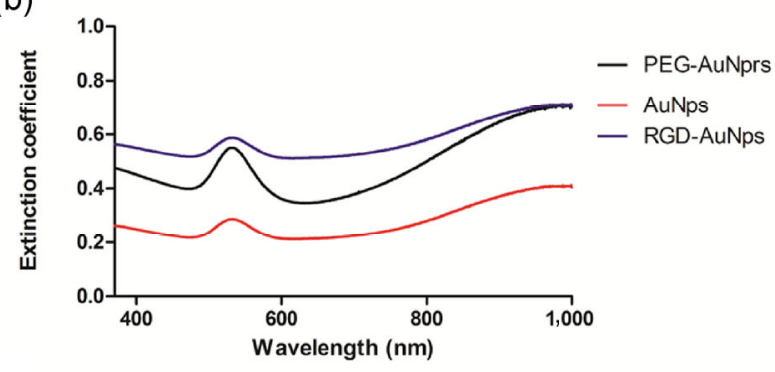

(d)

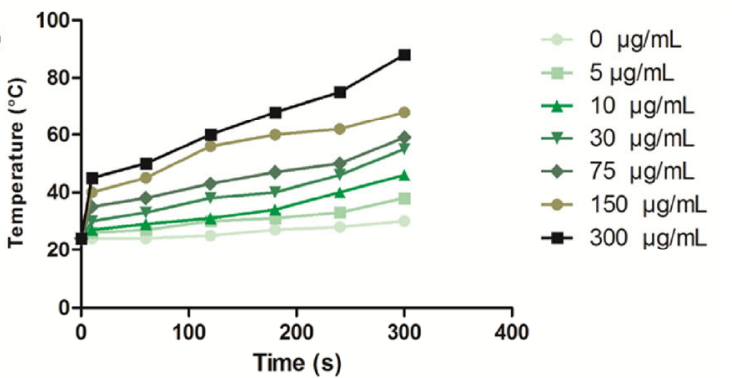

Figure 2 (a) TEM of AuNprs. (b) UV-vis-NIR spectra of AuNprs solution, PEGylated AuNprs, and RGD-AuNprs. (c) Dependence of PA signals on the AuNprs concentration. (d) Dependence of temperature on AuNprs concentration under the irradiation time of 5 min at $0.3 \mathrm{~W} / \mathrm{cm}^{2}$. 
PEGylated AuNprs can easily reach the cytoplasm (in $24 \mathrm{~h}$ ) of previously incubated MGC-803 cells (Fig. S2 in the ESM). White fluorescent dots observed in the cytoplasm of the cells indicate the intracellular uptake of the RGD-conjugated PEGylated AuNprs, whereas actin fibers are stained in red and Brcaa1-FITC in green and blue dots (Hoechst staining) indicate the nuclei of the cells.

The cytotoxicity of the antibody/peptide AuNprs was evaluated using the GES-1 cell line. An MTT assay was performed to assess the cell viability using increasing amounts of AuNprs without laser irradiation (Figs. S3 and S4 in the ESM). Increasing amounts (50, 100, 150, and $200 \mu \mathrm{g} / \mathrm{mL}$ ) of PEG-coated, RGD-coated PEGylated AuNprs, Brcaa1-antibodyconjugated PEGylated-AuNprs, as well as naked AuNprs were incubated with $5 \times 10^{3}$ cells for $24 \mathrm{~h}$. The Brcaa1-antibody-coated and RGD-coated PEGylated AuNprs showed nearly $100 \%$ cell viability with increasing concentration without light exposure. In addition, no noticeable cytotoxicity $(<10 \%)$ was observed for naked AuNprs.

Hydrodynamic light scattering (DLS) in water and zeta-potential data of all AuNprs-conjugates (naked AuNprs, PEG-AuNprs, RGD-AuNprs, and Brcaa1antibody-AuNprs) (Fig. S5 in the ESM), as well as the conjugation and quantification of Brcaa1 antibody on the surface of the nanoprisms (Fig. S6 in the ESM) was also performed in order to corroborate appropriate functionalization of the studied biomolecules.

In order to evaluate the cell structural integrity, MGC-803 cells incubated with RGD-coated PEGylated AuNprs were irradiated under $100 \mathrm{~mW}, 980 \mathrm{~nm}$ laser power for $3 \mathrm{~min}$. The cells were stained with Trypan Blue and imaged by optical microscopy. Although the cell morphology at this magnification remained normal, an increase in membrane permeability in response to Trypan Blue (vital stain used to selectively color dead cells only) due to the thermal effects of NPs was observed only for cells incubated with RGD-coated PEGylated AuNprs, when compared to control cells without exposure (Fig. S7 in the ESM).

In order to evaluate the full potential of the functionalized AuNprs for in vivo tumor targeting and angiography, an in vivo murine orthotopic model of gastric cancer was built. The tumor accumulation was characterized in MGC803 bearing mice by taking photoacoustic images after tail-vein administration of RGD-coated PEGylated AuNprs at a concentration of $1 \mathrm{mg} / \mathrm{mL}$. The best signal to noise ratio at a wavelength of $710 \mathrm{~nm}$ was selected and scanned. Using OsiriX software analysis, tumor vascular imaging was achieved via image acquisition for maximum density projection (MIP). Figure 3(a) shows in vivo photoacoustic images of subcutaneous transplanted tumors and sequential photoacoustic MIP frames of tumor blood vessels before and 1,3, and $6 \mathrm{~h}$ after tail-vein injection of RGD-coated PEGylated AuNprs. Using the conventional Nexus 128 system, imaging the tumor vasculature and the blood vessels could only be achieved through endogenous injection of a contrast agent, such as hemoglobin or deoxyhemoglobin. In addition, the tumor edges could roughly be imaged in the outermost region of the tumor (i.e. closest to the skin layer). The interior of the blood vessels was not visible. After tail-vein injection of RGD-coated PEGylated AuNprs, the blood vessels at the tumor site were clearly imaged, with an enhancement of the photoacoustic signal in a time-dependent manner (Figs. 3(b)-3(d)). In addition, RGD peptide-specific targeting of the tumor angiogenesis could be observed
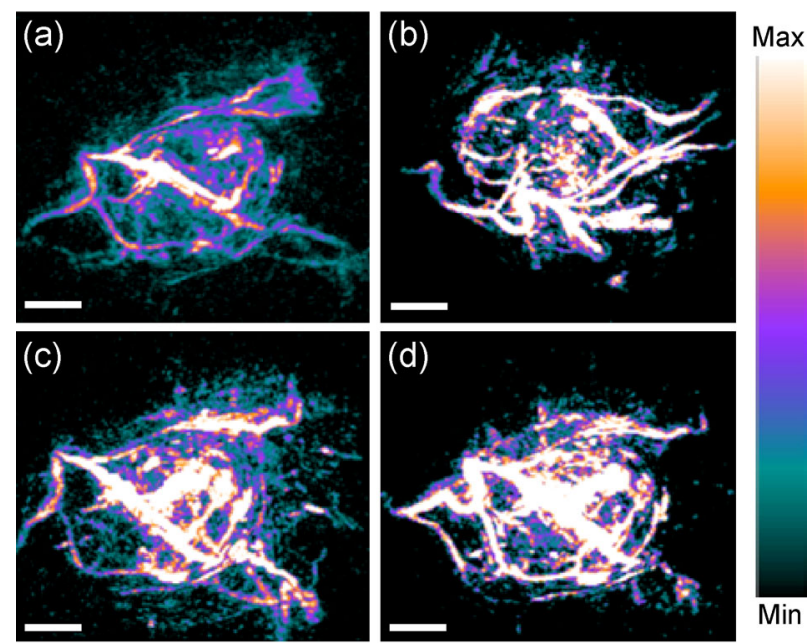

Figure 3 In vivo photoacoustic imaging study of subcutaneous transplanted tumors. (a)-(d) Sequential PA MIP frames after tailinjection with RGD-AuNprs. (a) Before injection with RGDAuNprs, (b) injection with RGD-AuNprs after $1 \mathrm{~h}$, (c) injection with RGD-AuNprs after $3 \mathrm{~h}$, (d) injection with RGD-AuNprs after $6 \mathrm{~h}$. Scale bars: $1 \mathrm{~mm}$. 
using the optoacoustic imaging of solid tumors to monitor angiogenesis and cancer progression. In fact, $4 \mathrm{~h}$ after injection, the prepared RGD-conjugated PEGylated AuNprs accumulated at the tumor site, as observed in the 3D PAI images of the tumors (Fig. S8 in the ESM). Moreover, the thermal effect of RGDconjugated PEGylated NPs could be exploited to treat cancer-diseased cells [23].

In order to evaluate the in situ functional targeting and imaging of gastric carcinoma, a second mouse model and a different functionalization of AuNprs were used. A gastric-cancer orthotopic mouse tumor model was developed and Brcaa1 antibody-conjugated PEGylated AuNprs (PEG/Brcaa1-AuNprs) were produced. First, the MGC-803 cell line was used to build the subcutaneous tumor model in nude mice. After the formation of the tumor tissue, the tumor tissue stability in nude mice was passaged three more times in order to access and insert fresh tumor tissue blocks in nude stomach via an $\mathrm{OB}$ adhesive construction of orthotopic gastric tumor transplantation. The OB glue paste technique is commonly used to establish nude mouse human gastric cancer orthotopic transplantation models. The OB glue paste technique is easy to perform and the biological behaviors of the nude mice human gastric cancer orthotopic transplantation models established with this technique are similar to the natural processes of growth and metastasis of human gastric cancer [24]. After establishing the orthotopic model, the blood supply and stomach tissue can easily be built. Rapid tumor growth in mice can be maintained for about four weeks. Figures S9(a) and S9(b) in the ESM show the orthotopic tumor mass model after four weeks, in which the blood supply and tumor volume significantly increase compared with the primary surgery to establish the tumor model (Fig. S9(a) in the ESM). Four weeks after the OB surgery, mice were tail-vein injected with PEG/Brcaa1-AuNprs and photoacoustic images were taken. Figure 4 and Fig. S10 in the ESM show the results for the photoacoustic imaging of mice without (Fig. 4(a) and Movie S1 in the ESM) and with intravenous injection of PEG-AuNprs after $24 \mathrm{~h}$ (Fig. 4(b) and Movie S2 in the ESM). As shown in Fig. 4(a), the MIP of the photoacoustic signal was weak and chaotic with a major interference from hemoglobin when there was no injection of contrast agent. In contrast, when injected with PEG/Brcaa1AuNprs, the tumor image improved significantly and imaging of the surface blood vessels was possible (Fig. 4(b) and Movie S2 in the ESM). At this time (24 h), no signal differences or levels of development could be distinguished between the various body organs, and therefore, the tumor location could not be determined to accomplish functional imaging.

Nevertheless, $24 \mathrm{~h}$ after injection of PEG/Brcaa1AuNprs, a significant difference between blood vessels, organs, and the tumor site was observed (Fig. 4(c) and Movie S3 in the ESM). Surgical scars (showing an " $X$ " shape), surgical nylon thread, and the gastric site (Fig. 4(c)) were easily displayed in photoacoustic imaging of PEG/Brcaa1-AuNprs treated mice. In addition, the signal from the gastric tissue site was more intense than the signal of surrounding organs such as the liver, proving that the PEG/Brcaa1-AuNprs effectively targets the gastric tumor site.

The shape and appearance of the mouse gastric tissue anatomy is depicted in Figs. 4(e) and 4(f). Gastric tumor tissues of mice treated with PEG/Brcaa1-AuNprs were stained using Brcaa1 immunofluorescent antibody only. Figs. 4(g)-4(i) show immunofluorescence staining of Brcaa1 in pathological tumor tissue using FITClabeled goat anti-mouse secondary antibody, confirming the specificity of the PEG/Brcaa1-AuNprs targeting.

These data prove that photoacoustic imaging can be used to overcome some of the disadvantages (i.e. radioactive conditions and poor resolution in soft tissues from CT/X-ray, poor resolution with deep penetration from NIR fluorescence imaging, or speckles of optical coherence tomography) of imaging modalities, and penetrate deeper with high-resolution imaging. Photoacoustic imaging in cancer detection has been mainly focused on melanoma models, subcutaneous tumor xenograft transplantation models, or in vitro lymphangiography [13]; however, detection is still confined to superficial parts of the body. Here, we could achieve in situ effective detection of tumor targeting with concomitant deep and high-resolution photoacoustic imaging of the tumor site. Most importantly, we could improve the visualization of surface acoustic imaging of diseased tissues, thus advancing the detection of internal organs of the targeted lesion. This method is not only capable of distinguishing the 

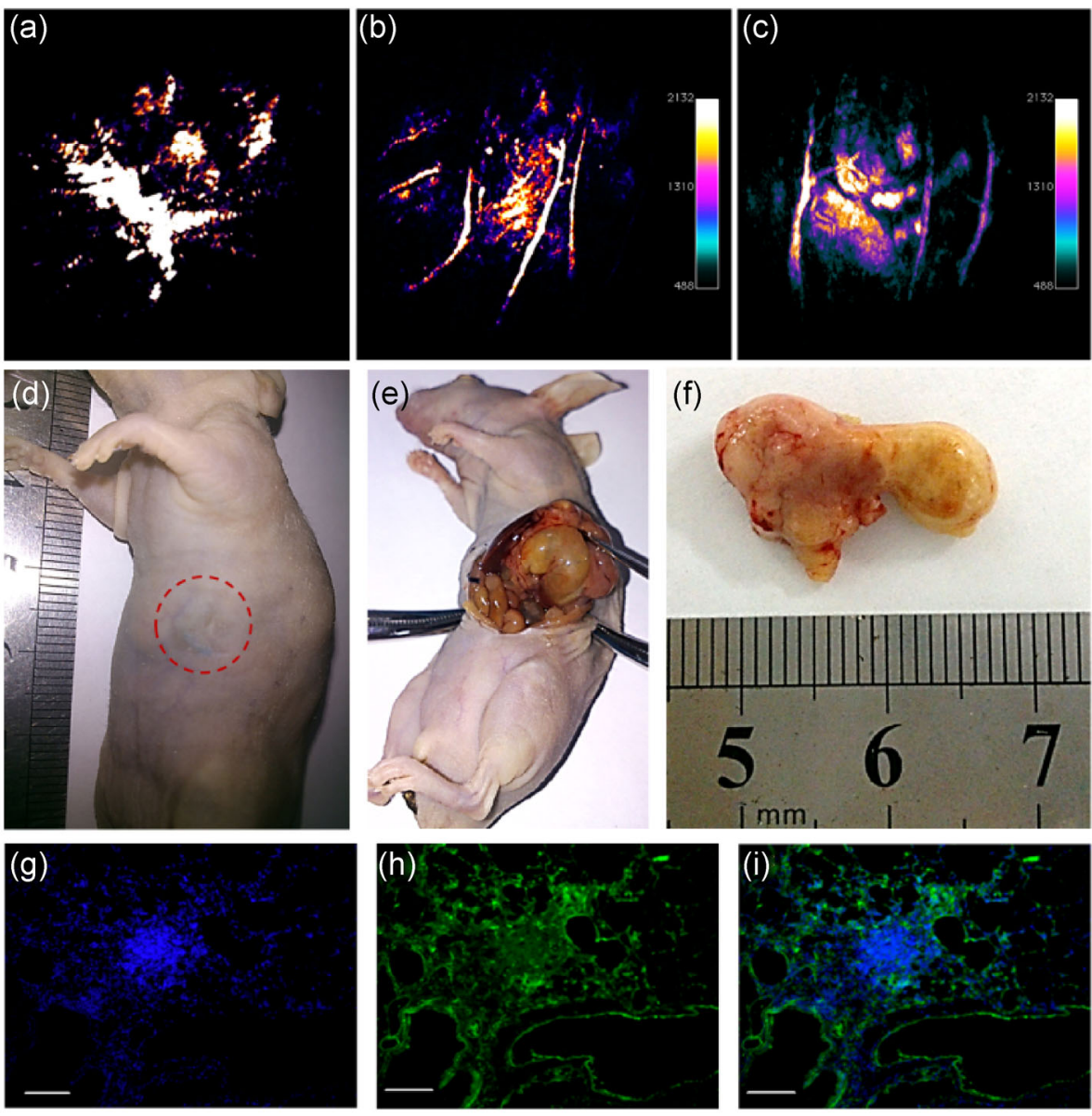

Figure 4 Representative photoacoustic imaging of gastric orthotopic tumor after injection of Brcaa1-AuNprs. (a) No injection of probes. (b) Photoacoustic imaging of gastric orthotopic tumor with injection of AuNprs after $24 \mathrm{~h}$. (c) In situ targeting photoacoustic imaging using intravenously injected PEG/Brcaa1-AuNprs after 48 h. (d) Image of scar and black surgical nylon thread. (e) and (f) Anatomical images of the localized gastric cancer tumor. (g)-(i) Immunohistochemical staining of tumor section from in situ gastric cancer tumor model. (g) DAPI staining of gastric tumor slides. (h) Immunofluorescence staining of Brcaal in human gastric tumor. (i) Merged image. Scale bar: $100 \mu \mathrm{m}$.

different organs, but also of achieving structural imaging of the tumor site and maximizing the signal to achieve functional imaging. Our method is now able to fully reflect the photoacoustic contrast advantage in cancer imaging.

In order to validate these results, PEG/RGD-coated AuNprs were used in X-ray and PEG/Brcaa1-coated AuNprs in CT imaging. Owing to its high atomic number and its absorption of X-rays, Au is often used in X-ray and CT imaging. In fact, many studies have shown that targeted Au NPs can increase the resolution at the tumor site, but with significantly higher concentrations of contrast agents. Here, we used $\sim 5 \mathrm{mg} / \mathrm{kg} \mathrm{Au}$ in the mouse model, although CT based on Au NPs usually requires $40-50 \mathrm{mg} / \mathrm{kg}$ Au for in vivo tumor imaging $[25,26]$. Therefore, PEG/RGD-coated AuNprs $(1 \mathrm{mg} / \mathrm{mL}$ in $100 \mu \mathrm{L})$ and PEG/Brcaa1-coated AuNprs $(1 \mathrm{mg} / \mathrm{mL}$ in $100 \mu \mathrm{L})$ were intravenously injected in mice bearing gastric tumors, and X-ray and CT detection was performed. As shown in Fig. 5, X-ray imaging of the control group (no NP treatment) showed no effect on the tumor site (Fig. 5(a)), whereas the X-ray image with $1 \mathrm{mg} / \mathrm{mL}$ of PEG/RGD-coated AuNprs had a significantly enhanced signal (Fig. 5(b)). The same applies for CT imaging, in which PEG/ Brcaa1-AuNprs $(1 \mathrm{mg} / \mathrm{mL}$ in $100 \mu \mathrm{L})$ were also intravenously injected in mice. The CT image with $1 \mathrm{mg} / \mathrm{mL}$ of PEG/Brcaa1-AuNprs showed no significant difference in the in situ gastric portion (Fig. 5(d)) when compared to the control group (Fig. 5(c)). 
(a)
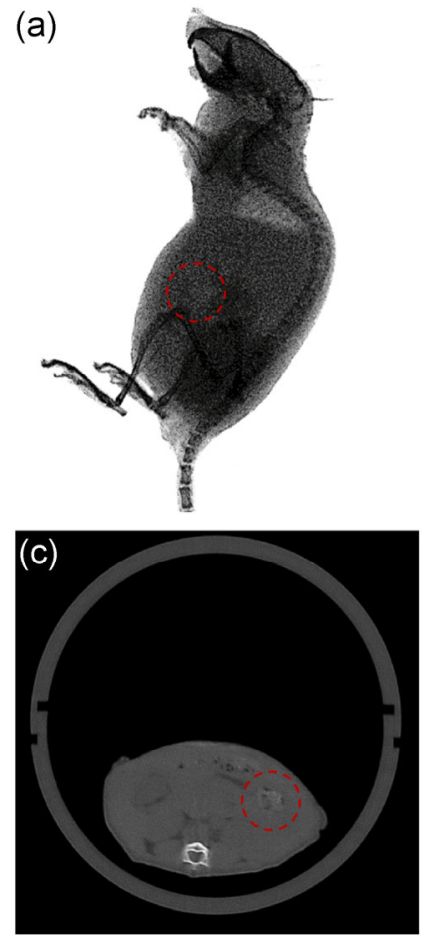

(b)
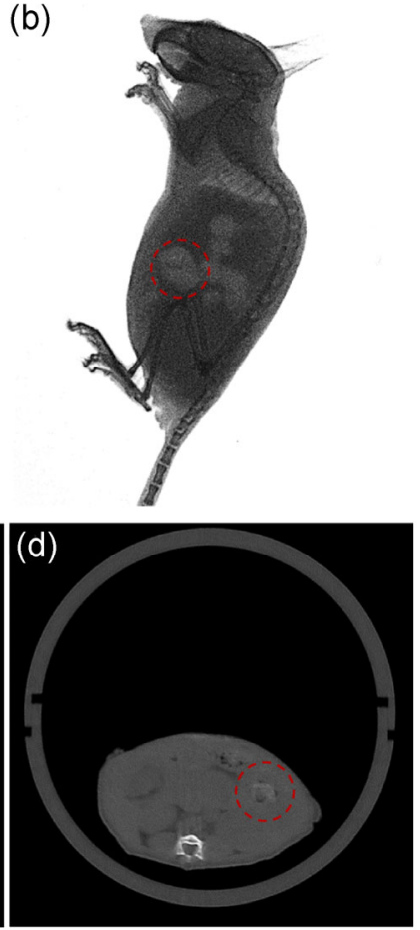

Figure 5 X-ray and micro CT imaging. (a) X-ray image of control group (no NP treatment). (b) X-ray image with $1 \mathrm{mg} / \mathrm{mL}$ of PEG/RGD-coated AuNprs. (c) CT image of control group. (d) CT image with $1 \mathrm{mg} / \mathrm{mL}$ of Brcaa1-AuNprs.

These data show that using a single-dose injection probe, photoacoustic imaging in a subcutaneous tumor model using PEG/RGD-coated AuNprs (see Fig. 3) and an in situ tumor model using PEG/Brcaa1-coated AuNprs (see Fig. 4) can achieve better sensitivity and resolution results than conventional $\mathrm{X}$-ray and $\mathrm{CT}$ imaging with the same dose of Au NPs.

In order to evaluate the localized hyperthermia effect mediated by the functionalized AuNprs, a subcutaneous gastric-cancer xenograft mice model was used. Mice were injected with $100 \mu \mathrm{L}$ of $1 \mathrm{mg} / \mathrm{mL}$ PEG/RGD-coated AuNprs via tail-vein injection. A $980 \mathrm{~nm}$ NIR laser and a high-definition digital infrared thermal imaging system were used for thermal imaging $2 \mathrm{~h}$ after injection (Fig. 6). The tumor was exposed to the $980 \mathrm{~nm}$ laser at $2 \mathrm{~h}$ post-injection $\left(0.3 \mathrm{~W} / \mathrm{cm}^{2}\right.$, $5 \mathrm{~min}$ ). Figure 6(a) represents the temperature variation at the tumor site from 0 to $5 \mathrm{~min}$ after laser irradiation. Figure 6(a1) represents a thermal image of the representative MGC-803 tumor-bearing mouse before the laser is turned on, when the skin surface temperature is only $25.9^{\circ} \mathrm{C}$. After irradiation for 100 (Fig. 6(a2)),
150 (Fig. 6(a3)), and $200 \mathrm{~s}$ (Fig. 6(a4)), the average temperatures at the tumor site after radiation are 40.7, 57.6, and $71.1^{\circ} \mathrm{C}$, respectively. Figure $6(\mathrm{~b})$ shows the hyperthermia in vivo assay measuring temperature variation in the control (no NP exposure) and experimental (injection of $100 \mu \mathrm{L}$ of $1 \mathrm{mg} / \mathrm{mL}$ PEG/RGDcoated AuNprs) groups. In each image, the average of seven temperature measurement points was taken (six points and one point in the center of the circumference selection). The temperature at the tumor site in the experimental group (PEG/RGD-coated AuNprs) reached a peak of $75^{\circ} \mathrm{C}$ after $200 \mathrm{~s}$ of exposure, showing significant thermal effects when compared to the control group, where the highest temperature is $30{ }^{\circ} \mathrm{C}$ (Figs. 6(b) and 6(c)). Figures 6(c1) and 6(c2) represent the local tumor morphology after localized hyperthermia of the control and experimental groups, respectively. The tissue without treatment (Fig. 6(c1)) had a normal appearance of intact skin, whereas the tissue after irradiation of the tumor exhibited a dark color (Fig. 6(c2)). Comparative results from photoacoustic imaging experiments showed deformation of the blood vessels surrounding the tumor tissue after hyperthermia therapy with PEG/RGD-coated AuNprs (Fig. 5(d)), while the control group at the same irradiation dose showed no significant change in tumor blood vessels (Fig. 6(d)). These results confirm the high efficiency of PEG/RGD-AuNprs and PEG/ Brcaa1-AuNprs as a combinatorial-targeted therapy in thermal imaging and therapy.

The contribution to mice survival from localized hyperthermia effects at the tumor site using PEG/ RGD-AuNprs was assessed by monitoring survival after 150 days. Animals treated with PEG/RGD-AuNprs survived significantly longer (Fig. 7(a)) than the control groups before and after therapy and PEG/RGDAuNprs group without therapy. The trends in prolonged survival from PEG/RGD-AuNprs after therapy treatment indicate that the observed survival extension of more than $85 \%$ could be attributed to the destruction of gastric cancer cells via localized hyperthermia.

Moreover, we hypothesized that the increase in mice survival using PEG/RGD-AuNprs after therapy would also enhance the likelihood of observing a tumor size reduction. Therefore, monitoring the change in tumor size as a function of time after treatment with 
(a)
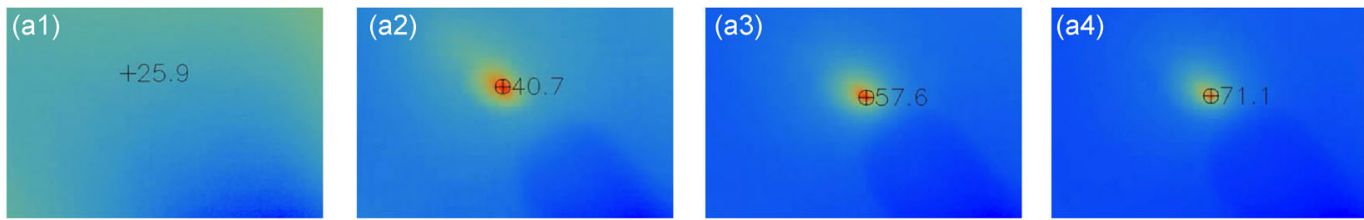

(b)
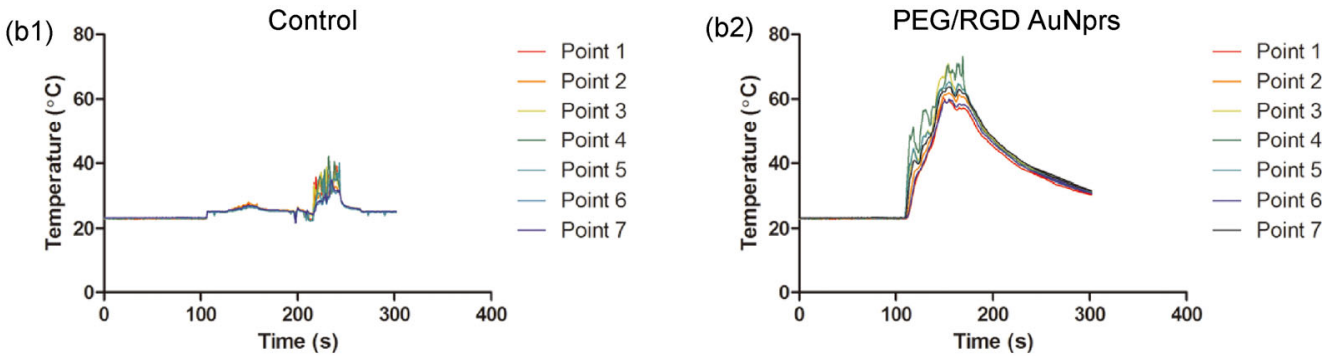

(c)
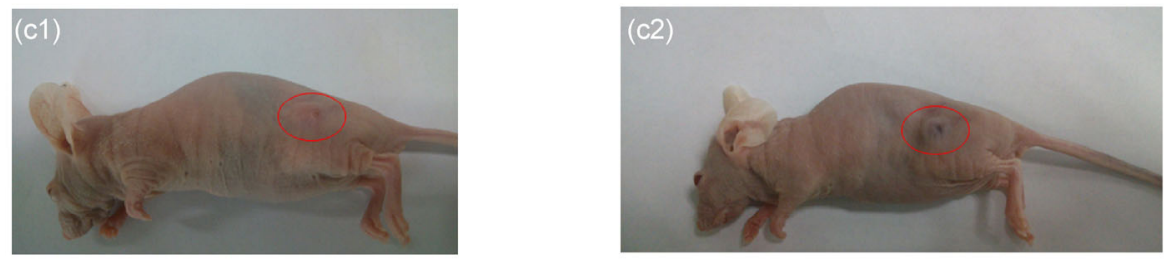

(d)

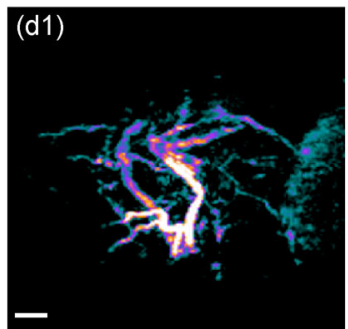

Control before therapy

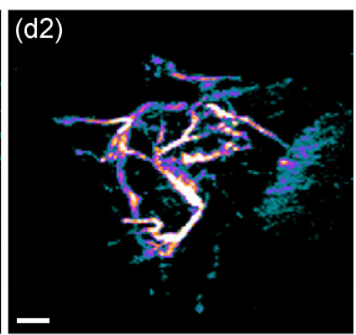

Control after therapy

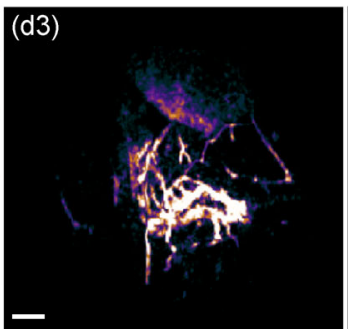

PEG/RGD AuNprs

before therapy

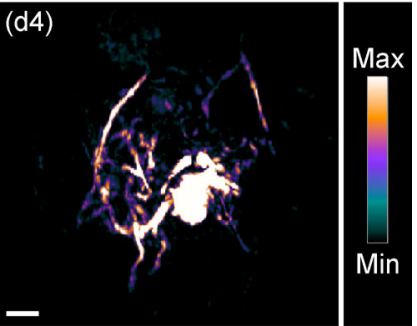

PEG/RGD AuNprs after therapy

Figure 6 In vivo photothermal therapy study using intravenously injected RGD-AuNprs. (a) Thermal images of representative MGC-803 tumor-bearing mouse exposed to $980 \mathrm{~nm}$ laser $\left(0.3 \mathrm{~W} / \mathrm{cm}^{2}\right)$ for $3 \mathrm{~min}$ at $1 \mathrm{~h}$ post-injection of RGD-AuNprs. (b) Tumor heating curves of the PBS and RGD-AuNprs injected mice groups. (c) Localized tumor images of mice after 3 min exposure to laser. (c1): control group, (c2): experimental group. (d) Photoacoustic image of angiography for control group injected with PBS and for experimental group injected with PEG/RGD-coated AuNprs monitoring before and after tumor therapy.

PEG/RGD-AuNprs associated with laser application revealed a significant decrease in tumor growth $(P<$ 0.005) at 27 days post AuNprs treatment (Fig. 7(b)) with a single-dose injection. After 35 days, animals treated with PEG/RGD-AuNprs after hyperthermia therapy show a tumor size of $22.1 \% \pm 7.6 \%$, compared to $100 \%$ $\pm 15.1 \%$ and $80.1 \% \pm 14.6 \%$ for control groups before and after treatment, respectively, and $90.0 \% \pm 9.6 \%$ for PEG/RGD-AuNprs before therapy.

The improved outcome in gastric tumor-bearing mice receiving PEG/RGD-AuNprs treatment strongly supports the extraordinary potential of these NPs as adjuvant agents to anticancer therapies.
The safety and biodistribution of PEG/RGD-AuNprs was confirmed by ex vivo organ inductively coupled plasma mass spectrometry (ICP-MS) for $6 \mathrm{~h}$ and 14 days after injection of the probes. The results show that $6 \mathrm{~h}$ after injection, PEG/RGD-AuNprs were mainly accumulated in the liver, kidney, lung, and tumor. After 14 days, the Au content decreased in most of the tissues and organs, especially in the heart and kidney (Fig. 7(c)).

To validate the safety assessment, 14 days after injection of PEG/RGD-AuNprs, organs were harvested from mice and H\&E stained for routine pathological analysis (Fig. 7(d)). H\&E staining shows that in vivo 

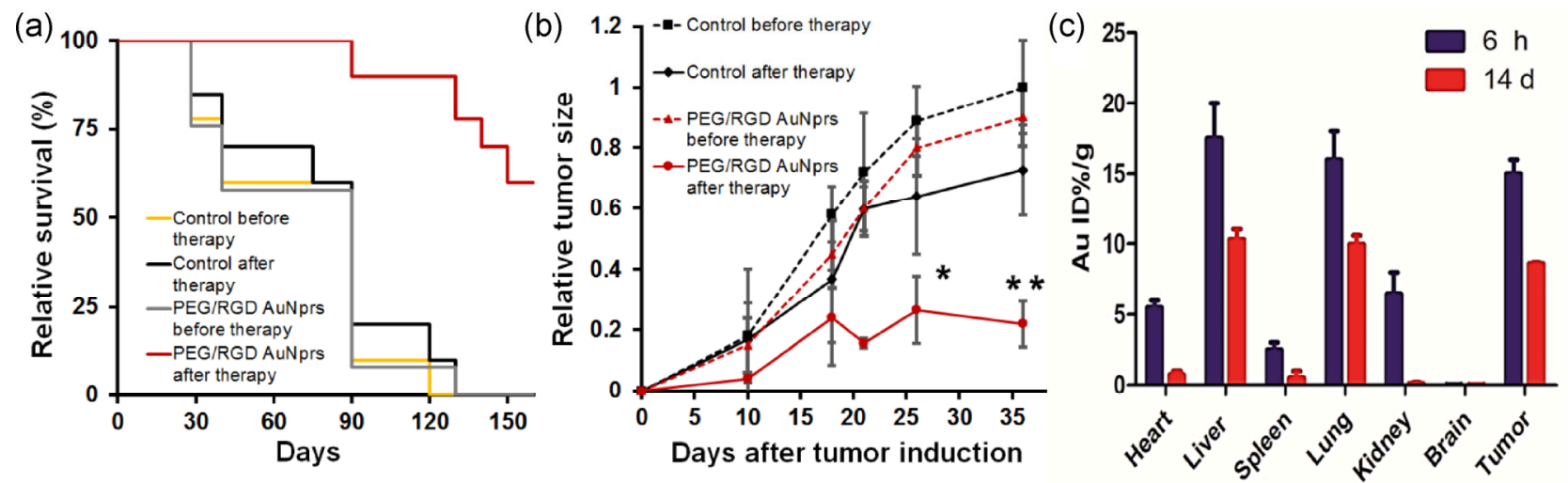

(c)
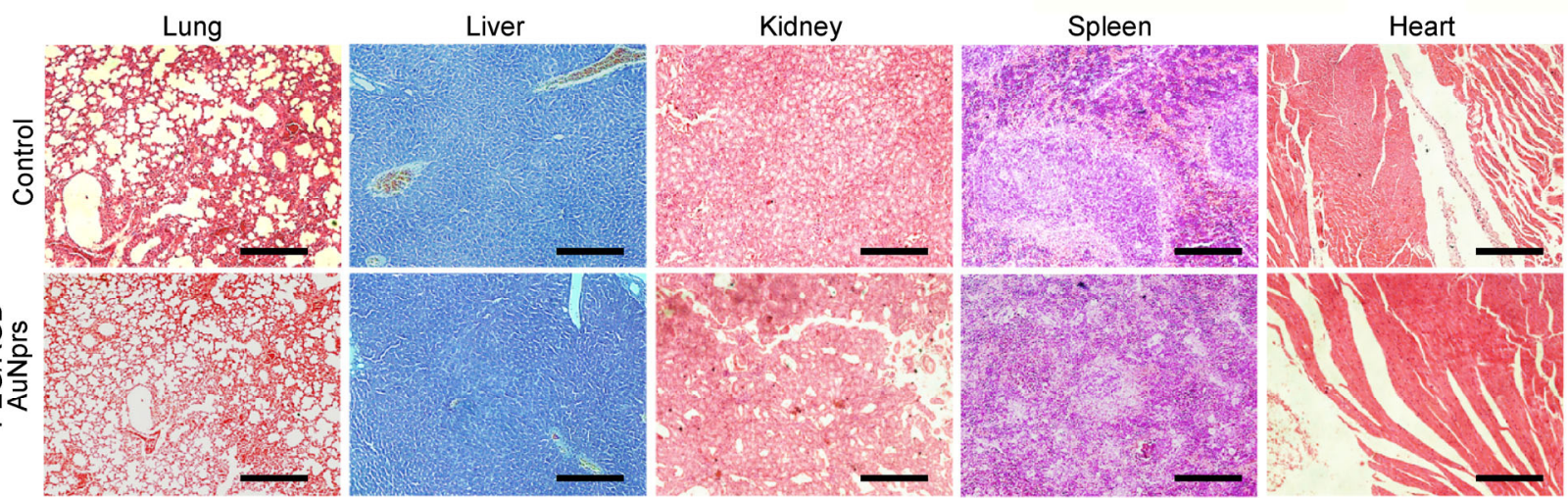

Figure 7 Therapeutic outcome following treatment with PEG/RGD AuNprs in subcutaneous transplanted gastric tumors. (a) KaplanMeier survival curves during 150 days of control mice (no NP treatment) and for PEG/RGD AuNprs before and after localized hyperthermia therapy. (b) Relative tumor size in mice bearing gastric tumor xenografts treated with PEG/RGD AuNprs before and after localized hyperthermia therapy and compared to control groups. (c) ICP-MS of Au content at $6 \mathrm{~h}$ and 14 days after injection with PEG/RGD-AuNprs $(n=3)$. (d) H\&E stained tissue sections of the organs (i.e. lung, liver, kidney, spleen, heart) collected from mice 20 days after intravenous injection of a single dose of PEG/RGD-AuNprs. Bar: $50 \mu \mathrm{m}$.

administration of PEG/RGD-AuNprs did not damage to several organs (i.e. lung, liver, kidney, spleen, or heart) when compared to the control group (without AuNpr administration). The lungs showed a clear and complete alveolar structure, a uniform distribution of the nucleus and cytoplasm was observed in liver tissue, the kidney tubules and glomeruli showed no obvious lesions, and the spleen showed normal white and red pulp distribution. Moreover, a normal cardiac morphology and intracellular free grainy was observed in the heart.

\section{Conclusions}

Traditional imaging techniques are frequently limited by narrow penetration, low sensitivity, low specificity, and poor spatial resolution. Hybrid modalities such as optoacoustic imaging, an emerging molecular imaging modality, have greatly contributed to improving most of these limitations.

In summary, we developed a hybrid approach for both photoacoustic angiography and localized hyperthermia using antibody/peptide AuNprs in a subcutaneous and an orthotopic murine gastric carcinoma model. This system proved to be highly efficient for in situ photoacoustic imaging, with high-resolution of blood vessels at the tumor site and an enhancement of the photoacoustic signal coupled to localized hyperthermia. With a single-dose injection, these smart antibody/peptide AuNprs are responsible for an extensive tumor size reduction and increase in mice survival after localized hyperthermia treatment.

This is the first proof-of-concept using two AuNprs probes as high sensitivity contrasts and therapeutic 
agents for in vivo tumor detection and inhibition, which can be used as an efficient and safe nanotheranostic platform. Taken together, the results reported herein establish the mechanism of action of AuNprs in both imaging and targeting approaches, as well as the relationship between hyperthermia time, intensity, and efficacy in an in vivo mouse model, which will translate in a very meaningful way to cancer clinical research.

\section{Acknowledgements}

This work was supported by the National Basic Research Program of China (No. 2015CB931802), National Natural Science Foundation of China (Nos. 81225010, 81327002, 31170961, 20771075, and 20803040), the National High-tech R\&D Program of China (No. 2014AA020700), and Special project for nanotechnology from Shanghai (Nos. 13NM1401500 and 15DZ2252000).

Electronic Supplementary Material: Supplementary material is available in the online version of this article at http://dx.doi.org/10.1007/s12274-016-0996-y.

\section{References}

[1] Feynman, R. P. There's plenty of room at the bottom. Eng. Sci. 1960, 23, 22-36.

[2] Bao, C. C.; Chen, L.; Wang, T.; Lei, C.; Tian, F. R.; Cui, D. X.; Zhou, Y. One step quick detection of cancer cell surface marker by integrated nife-based magnetic biosensing cell cultural chip. Nano-Micro Lett. 2013, 5, 213-222.

[3] Ferrari, M. Cancer nanotechnology: Opportunities and challenges. Nat. Rev. Cancer 2005, 5, 161-171.

[4] Schroeder, A.; Heller, D. A.; Winslow, M. M.; Dahlman, J. E.; Pratt, G. W.; Langer, R.; Jacks, T.; Anderson, D. G. Treating metastatic cancer with nanotechnology. Nat. Rev. Cancer 2011, 12, 39-50.

[5] Conde, J.; Doria, G.; Baptista, P. Noble metal nanoparticles applications in cancer. J. Drug Deliv. 2011, 2012, Article ID 751075 .

[6] Lee, J.-H.; Huh, Y.-M.; Jun, Y.-W.; Seo, J.-W.; Jang, J.-T.; Song, H.-T.; Kim, S.; Cho, E.-J.; Yoon, H.-G.; Suh, J.-S. Artificially engineered magnetic nanoparticles for ultrasensitive molecular imaging. Nat. Med. 2007, 13, 95-99.

[7] Pelaz, B.; Grazu, V.; Ibarra, A.; Magen, C.; del Pino, P.; de la Fuente, J. M. Tailoring the synthesis and heating ability of gold nanoprisms for bioapplications. Langmuir 2012, 28, 8965-8970.

[8] Ambrosone, A.; del Pino, P.; Marchesano, V.; Parak, W. J.; de la Fuente, J. M.; Tortiglione, C. Gold nanoprisms for photothermal cell ablation in vivo. Nanomedicine 2014, 9, 1913-1922.

[9] Bao, C. C.; Beziere, N.; del Pino, P.; Pelaz, B.; Estrada, G.; Tian, F. R.; Ntziachristos, V.; de la Fuente, J. M.; Cui, D. X. Gold nanoprisms as optoacoustic signal nanoamplifiers for in vivo bioimaging of gastrointestinal cancers. Small 2013, 9, 68-74.

[10] Conde, J.; Rosa, J.; Lima, J. C.; Baptista, P. V. Nanophotonics for molecular diagnostics and therapy applications. Int. J. Photoenergy 2012, 2012, Article ID 619530.

[11] Yang, X. M.; Skrabalak, S. E.; Li, Z.-Y.; Xia, Y. N.; Wang, L. V. Photoacoustic tomography of a rat cerebral cortex in vivo with Au nanocages as an optical contrast agent. Nano Lett. 2007, 7, 3798-3802.

[12] Eghtedari, M.; Oraevsky, A.; Copland, J. A.; Kotov, N. A.; Conjusteau, A.; Motamedi, M. High sensitivity of in vivo detection of gold nanorods using a laser optoacoustic imaging system. Nano Lett. 2007, 7, 1914-1918.

[13] Song, K. H.; Kim, C.; Maslov, K.; Wang, L. V. Noninvasive in vivo spectroscopic nanorod-contrast photoacoustic mapping of sentinel lymph nodes. Eur. J. Radiol. 2009, 70, 227-231.

[14] Hildebrandt, B.; Wust, P.; Ahlers, O.; Dieing, A.; Sreenivasa, G.; Kerner, T.; Felix, R.; Riess, H. The cellular and molecular basis of hyperthermia. Crit. Rev. Oncol./Hematol. 2002, 43, 33-56.

[15] Huang, X. H.; El-Sayed, I. H.; Qian, W.; El-Sayed, M. A. Cancer cell imaging and photothermal therapy in the nearinfrared region by using gold nanorods. J. Am. Chem. Soc. 2006, 128, 2115-2120.

[16] Han, J. S.; Zhang, J. J.; Yang, M.; Cui, D. X.; de la Fuente, J. M. Glucose-functionalized Au nanoprisms for optoacoustic imaging and near-infrared photothermal therapy. Nanoscale 2016, 8, 492-499.

[17] Cui, D.; Jin, G.; Gao, T. W.; Sun, T.; Tian, F.; Estrada, G. G.; Gao, H.; Sarai, A. Characterization of BRCAA1 and its novel antigen epitope identification. Cancer Epidemiol. Biomarkers Prev. 2004, 13, 1136-1145.

[18] Chen, X. Y. Multimodality imaging of tumor integrin $\alpha v \beta 3$ expression. Mini Rev. Med. Chem. 2006, 6, 227-234.

[19] Montet, X.; Montet-Abou, K.; Reynolds, F.; Weissleder, R.; Josephson, L. Nanoparticle imaging of integrins on tumor cells. Neoplasia 2006, 8, 214-222.

[20] Li, Z.; Huang, P.; Zhang, X.; Lin, J.; Yang, S.; Liu, B.; Gao, F.; Xi, P.; Ren, Q.; Cui, D. RGD-conjugated dendrimer- 
modified gold nanorods for in vivo tumor targeting and photothermal therapy. Mol. Pharm. 2009, 7, 94-104.

[21] Wang, K.; Ruan, J.; Qian, Q. R.; Song, H.; Bao, C. C.; Zhang, X. Q.; Kong, Y. F.; Zhang, C. L.; Hu, G. H.; Ni, J. et al. BRCAA1 monoclonal antibody conjugated fluorescent magnetic nanoparticles for in vivo targeted magnetofluorescent imaging of gastric cancer. J Nanobiotechnology 2011, 9, 23.

[22] Li, C.; Ji, Y.; Wang, C.; Liang, S. J.; Pan, F.; Zhang, C. L.; Chen, F.; Fu, H. L.; Wang, K.; Cui, D. X. BRCAA1 antibody- and Her2 antibody-conjugated amphiphilic polymer engineered $\mathrm{CdSe} / \mathrm{ZnS}$ quantum dots for targeted imaging of gastric cancer. Nanoscale Res. Lett. 2014, 9, 244.

[23] Nie, L. M.; Wang, S. J.; Wang, X. Y.; Rong, P. F.; Ma, Y.; Liu, G.; Huang, P.; Lu, G. M.; Chen, X. Y. In vivo volumetric photoacoustic molecular angiography and therapeutic monitoring with targeted plasmonic nanostars. Small 2014, 10, 1585-1593.

[24] Shi, J.; Wei, P.-K.; Zhang, S.; Qin, Z.-F.; Li, J.; Sun, D.-Z.; Xiao, Y.; Yu, Z.-H.; Lin, H.-M.; Zheng, G.-J. et al. OB glue paste technique for establishing nude mouse human gastric cancer orthotopic transplantation models. World J. Gastroenterol. 2008, 14, 4800-4804.

[25] Kim, D.; Park, S.; Lee, J. H.; Jeong, Y. Y.; Jon, S. Antibiofouling polymer-coated gold nanoparticles as a contrast agent for in vivo X-ray computed tomography imaging. $J$. Am. Chem. Soc. 2007, 129, 7661-7665.

[26] von Maltzahn, G.; Park, J.-H.; Agrawal, A.; Bandaru, N. K.; Das, S. K.; Sailor, M. J.; Bhatia, S. N. Computationally guided photothermal tumor therapy using long-circulating gold nanorod antennas. Cancer Res. 2009, 69, 3892-3900. 